VOL. $67(2003) \quad[1-14]$

\title{
IRREDUCIBLE DECOMPOSITION OF THE MAGNUS REPRESENTATION OF THE TORELLI GROUP
}

\author{
MASAAKI SUZUKI
}

In this paper we describe the irreducible decomposition of the Magnus representation of the Torelli group.

\section{INTRODUCTION}

Let $\Sigma_{g}$ be a closed oriented surface of genus $g$ and $\Sigma_{g, 1}$ be an oriented surface obtained from $\Sigma_{g}$ by removing an open disk. We denote by $\mathcal{M}_{g, 1}$ the mapping class group of $\Sigma_{g, 1}$ relative to the boundary, that is, the group of path components of the group of orientation preserving diffeomorphisms of $\Sigma_{g, 1}$ which restrict to the identity on the boundary. Let $\mathcal{I}_{g, 1}$ be the Torelli group of $\Sigma_{g, 1}$, namely the normal subgroup of $\mathcal{M}_{g, 1}$ consisting of all the elements which act on the homology of $\Sigma_{g, 1}$ trivially. Various results concerning the structure of the mapping class group and the Torelli group have been obtained (for example see $[5,7])$.

We investigate the Magnus representation of the Torelli group. The aim of this paper is to give the irreducible decomposition of this representation. The Magnus representations are defined for a wide class of subgroups of automorphism group of free groups (see [2] for details). For example, the classical Burau representation and the Gassner representation, for braid groups and pure braid groups respectively, belong to this class. The Magnus representation for the mapping class group $\mathcal{M}_{g, 1}$

$$
r: \mathcal{M}_{g, 1} \rightarrow G L\left(2 g ; \mathbb{Z}\left[\Gamma_{0}\right]\right)
$$

was studied in [6], where $\Gamma_{0}=\pi_{1}\left(\Sigma_{g, 1}\right)$. This mapping is not a group homomorphism. We restrict it to the Torelli group $\mathcal{I}_{g, 1}$ and reduce the coefficients to $\mathbb{Z}[H]$ which is induced by the Abelianisation $\mathfrak{a}: \Gamma_{0} \rightarrow H$, where $H=H_{1}\left(\Sigma_{g, 1} ; \mathbb{Z}\right)$. Then we obtain a homomorphism

$$
\bar{r}: \mathcal{I}_{g, 1} \longrightarrow G L(2 g ; \mathbb{Z}[H]) .
$$

Received 23rd October, 2001

The author would like to express his gratitude to Professor Shigeyuki Morita for helpful suggestions and encouragements. He also would like to thank Professor Nariya Kawazumi, Professor Teruaki Kitano and Professor Takayuki Morifuji for valuable discussions and advices.

Copyright Clearance Centre, Inc. Serial-fee code: 0004-9727/03 \$A2.00+0.00. 
We call this mapping $\bar{r}$ the Magnus representation of the Torelli group. In Section 2, we recall the definition of the Magnus representation of the Torelli group more precisely from [6].

Each of the Burau and Gassner representations has a 1-dimensional trivial subrepresentation. In Section 3, we show that the Magnus representation of the Torelli group also has a 1-dimensional trivial subrepresentation. In contrast to the classical cases, however, this 1-dimensional trivial subrepresentation is not a direct summand. In Section 4, we show that the quotient representation is reducible in our case. In fact, it has a $(2 g-2)$ dimensional irreducible subrepresentation. That is to say, we arrive at the following main result of this paper.

MAIN ThEOREM. For $g \geqslant 2$ there exsists a non-singular matrix $P \in G L(2 g ; R)$ such that for any element $\varphi \in \mathcal{I}_{g, 1}$

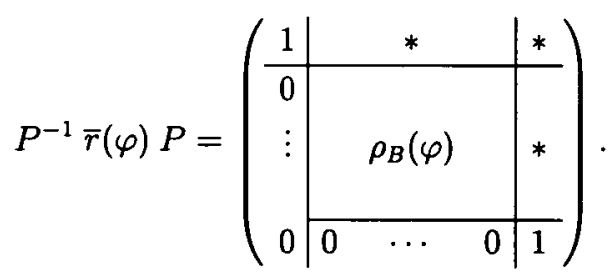

Moreover, $\rho_{B}$ is a $(2 g-2)$-dimensional irreducible representation of $\mathcal{I}_{g, 1}$.

Here $R=\mathbb{Z}\left[x_{i}^{ \pm 1}, y_{i}^{ \pm 1}, 1 /\left(1-y_{i}\right)\right](\supset \mathbb{Z}[H])$ where $x_{i}, y_{i}(i=1, \ldots, g)$ is a symplectic basis of $H$ obtained by Abelianising a system of generators $\alpha_{i}, \beta_{i}$ of $\Gamma_{0}$ as shown in Figure 1. We show that the representation $\rho_{B}$ is irreducible by making use of Formanek's technique used in [4] to determine whether the reduced Burau representation obtained by the complex specialisation is irreducible or not. His technique was also used in [1] to give a necessary and sufficient condition for the specialisation of the reduced Gassner representation to be irreducible.

In Section 6, we give some remarks and applications of the irreducible decomposition.

\section{Definition of the Magnus representation}

We denote by $\mathbb{Z}\left[\Gamma_{0}\right]$ the integral group ring of $\Gamma_{0}=\pi_{1}\left(\Sigma_{g, 1}\right)$. We fix a system of generators $\alpha_{1}, \ldots, \alpha_{g}, \beta_{1}, \ldots, \beta_{g}$ of the free group $\Gamma_{0}$ as shown in Figure 1. Let us simply write $\gamma_{1}, \ldots, \gamma_{2 g}$ for them.

Definition 2.1: We call the following mapping

$$
\begin{aligned}
r: \mathcal{M}_{g, 1} & \longrightarrow G L\left(2 g ; \mathbb{Z}\left[\Gamma_{0}\right]\right) \\
\varphi & \longmapsto\left(\frac{\partial \varphi\left(\gamma_{j}\right)}{\partial \gamma_{i}}\right)_{i, j}
\end{aligned}
$$




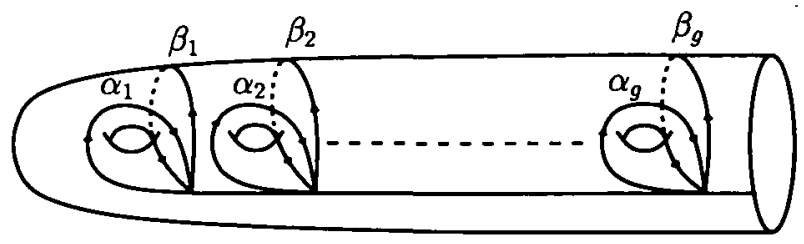

Figure 1: Generators of $\Gamma_{0}$

the Magnus representation for the mapping class group $\mathcal{M}_{g, 1}$. Here $\frac{\partial}{\partial \gamma_{i}}$ is the Fox derivation and ${ }^{-}: \mathbb{Z}\left[\Gamma_{0}\right] \rightarrow \mathbb{Z}\left[\Gamma_{0}\right]$ is the antiautomorphism induced by the mapping $\gamma \mapsto \gamma^{-1}$.

However, this mapping $r$ is not a group homomorphism.

Proposition 2.2. ([6]) For any two elements $\varphi, \psi \in \mathcal{M}_{g, 1}$, we have

$$
r(\varphi \psi)=r(\varphi) \cdot \varphi r(\psi)
$$

where ${ }^{\varphi} r(\psi)$ denotes the matrix obtained from $r(\psi)$ by applying the automorphism $\varphi$ : $\mathbb{Z}\left[\Gamma_{0}\right] \rightarrow \mathbb{Z}\left[\Gamma_{0}\right]$ on each entry.

We restrict this mapping $r$ to the Torelli group $\mathcal{I}_{g, 1}$ and reduce the coefficients to $\mathbb{Z}[H]$. Since the Torelli group $\mathcal{I}_{g, 1}$ acts trivially on $H$, we obtain a genuine homomorphism

$$
\bar{r}: \mathcal{I}_{g, 1} \rightarrow G L(2 g ; \mathbb{Z}[H]) .
$$

This is the definition of the Magnus representation of the Torelli group.

\section{Reducibility of the Magnus Representation of the Torelli group and}

\section{A QUOTIENT REPRESENTATION}

As is mentioned in [2], the classical Burau representation as well as the Gassner representation has a 1-dimensional trivial subrepresentation. The Magnus representation of the Torelli group has a similar property.

We define the vector $v$ as follows. Let $\zeta$ be a simple closed curve on $\Sigma_{g, 1}$ which is parallel to the boundary. We may regard $\zeta$ as an element of $\Gamma_{0}$.

$$
\zeta=\left[\beta_{g}, \alpha_{g}\right] \cdots\left[\beta_{1}, \alpha_{1}\right] \in \Gamma_{0}
$$

The vector $v$ is obtained by applying Fox derivations to the boundary curve $\zeta$. That is, we set

$$
\begin{aligned}
v & ={ }^{t}\left(\mathfrak{a}\left(\overline{\frac{\partial \zeta}{\partial \alpha_{1}}}\right) \cdots \mathfrak{a}\left(\overline{\frac{\partial \zeta}{\partial \alpha_{g}}}\right) \mathfrak{a}\left(\overline{\frac{\partial \zeta}{\partial \beta_{1}}}\right) \cdots \mathfrak{a}\left(\overline{\frac{\partial \zeta}{\partial \beta_{g}}}\right)\right) \\
& ={ }^{t}\left(-b_{1} \cdots-b_{g} a_{1} \cdots a_{g}\right)
\end{aligned}
$$

where $a_{i}, b_{i}$ are $1-\bar{x}_{i}, 1-\bar{y}_{i}$ respectively and $\bar{x}_{i}=x_{i}{ }^{-1}, \bar{y}_{i}=y_{i}{ }^{-1}$. 
Proposition 3.1. The Magnus representation of the Torelli group has a 1dimensional trivial subrepresentation. In fact, a 1-dimensional subspace spanned by the vector $v$ is invariant under the action of $\mathcal{I}_{g, 1}$.

Proof: For a given element $\varphi \in \mathcal{I}_{g, 1}$, we write simply

$$
\bar{r}(\varphi)=\left(\begin{array}{ccc}
m_{1,1} & \cdots & m_{1,2 g} \\
\vdots & \ddots & \vdots \\
m_{2 g, 1} & \cdots & m_{2 g, 2 g}
\end{array}\right) .
$$

Let $\tau_{\zeta}$ be the Dehn twist along a simple closed curve $\zeta$. By straightforward calculation, we obtain the following matrix.

$$
\bar{r}\left(\tau_{\zeta}\right)=I_{2 g}+{ }^{t}\left(-b_{1} \cdots-b_{g} a_{1} \cdots a_{g}\right) \cdot\left(a_{1} \cdots a_{g} b_{1} \cdots b_{g}\right)
$$

Here $I_{2 g}$ denotes the $2 g \times 2 g$ unit matrix. Therefore

$$
\begin{aligned}
\bar{r}\left(\varphi \tau_{\zeta}\right)= & \bar{r}(\varphi) \bar{r}\left(\tau_{\zeta}\right) \\
= & \left(\begin{array}{ccc}
m_{1,1} & \cdots & m_{1,2 g} \\
\vdots & \ddots & \vdots \\
m_{2 g, 1} & \cdots & m_{2 g, 2 g}
\end{array}\right) \\
& +\left(\begin{array}{ccc}
m_{1,1} & \cdots & m_{1,2 g} \\
\vdots & \ddots & \vdots \\
m_{2 g, 1} & \cdots & m_{2 g, 2 g}
\end{array}\right)\left(\begin{array}{c}
-b_{1} \\
\vdots \\
-b_{g} \\
a_{1} \\
\vdots \\
a_{g}
\end{array}\right)\left(\begin{array}{lllll}
a_{1} & \cdots & a_{g} b_{1} & \cdots & b_{g}
\end{array}\right) .
\end{aligned}
$$

On the other hand, we can easily compute the same matrix directly by the definition, because the action of $\varphi \tau_{\zeta} \in \mathcal{I}_{g, 1}$ on $\pi_{1}\left(\Sigma_{g, 1}\right)$ is given by

$$
\varphi \tau_{\zeta}\left(\gamma_{j}\right)=\left[\beta_{g}, \alpha_{g}\right] \cdots\left[\beta_{1}, \alpha_{1}\right] \varphi\left(\gamma_{j}\right)\left[\alpha_{1}, \beta_{1}\right] \cdots\left[\alpha_{g}, \beta_{g}\right] .
$$

For example, the Fox derivations of them are computed as

$$
\begin{aligned}
\frac{\partial\left(\varphi \tau_{\zeta}\left(\alpha_{j}\right)\right)}{\partial \alpha_{i}}=[ & \left.\beta_{g}, \alpha_{g}\right] \cdots\left[\beta_{i+1}, \alpha_{i+1}\right] \beta_{i} \\
& -\left[\beta_{g}, \alpha_{g}\right] \cdots\left[\beta_{i}, \alpha_{i}\right] \\
& +\left[\beta_{g}, \alpha_{g}\right] \cdots\left[\beta_{1}, \alpha_{1}\right] \frac{\partial \varphi\left(\alpha_{j}\right)}{\partial \alpha_{i}} \\
& +\left[\beta_{g}, \alpha_{g}\right] \cdots\left[\beta_{1}, \alpha_{1}\right] \varphi\left(\alpha_{j}\right)\left[\alpha_{1}, \beta_{1}\right] \cdots\left[\alpha_{i-1}, \beta_{i-1}\right] \\
& -\left[\beta_{g}, \alpha_{g}\right] \cdots\left[\beta_{1}, \alpha_{1}\right] \varphi\left(\alpha_{j}\right)\left[\alpha_{1}, \beta_{1}\right] \cdots\left[\alpha_{i-1}, \beta_{i-1}\right] \alpha_{i} \beta_{i} \bar{\alpha}_{i} .
\end{aligned}
$$


Since $a\left(\frac{\partial \varphi\left(\alpha_{j}\right)}{\partial \alpha_{i}}\right)=m_{i, j}$ and $\mathfrak{a}\left(\varphi\left(\alpha_{j}\right)\right)=x_{j}$, the $(i, j)$-component of the matrix $\bar{r}\left(\varphi \tau_{\zeta}\right)$ is expressed as

$$
\mathrm{a}\left(\overline{\frac{\partial\left(\varphi \tau_{\zeta}\left(\alpha_{j}\right)\right)}{\partial \alpha_{i}}}\right)=m_{i, j}-\left(1-\bar{y}_{i}\right)\left(1-\bar{x}_{j}\right) \quad i, j=1, \ldots, g .
$$

Similarly we have the following $(i, j=1, \ldots, g)$.

$$
\begin{aligned}
& \mathfrak{a}\left(\frac{\left.\overline{\frac{\partial\left(\varphi \tau_{\zeta}\left(\beta_{j}\right)\right)}{\partial \alpha_{i}}}\right)}{}=m_{i, g+j}-\left(1-\bar{y}_{i}\right)\left(1-\bar{y}_{j}\right)\right. \\
& \mathfrak{a}\left(\frac{\partial\left(\varphi \tau_{\zeta}\left(\alpha_{j}\right)\right)}{\partial \beta_{i}}\right)=m_{g+i, j}+\left(1-\bar{x}_{i}\right)\left(1-\bar{x}_{j}\right) \\
& \mathfrak{a}\left(\frac{\overline{\partial\left(\varphi \tau_{\zeta}\left(\beta_{j}\right)\right)}}{\partial \beta_{i}}\right)=m_{g+i, g+j}+\left(1-\bar{x}_{i}\right)\left(1-\bar{y}_{j}\right)
\end{aligned}
$$

Thus we obtain the matrix $\bar{r}\left(\varphi \tau_{\zeta}\right)$

$$
\bar{r}\left(\varphi \tau_{\zeta}\right)=\left(\begin{array}{ccc}
m_{1,1} & \cdots & m_{1,2 g} \\
\vdots & \ddots & \vdots \\
m_{2 g, 1} & \cdots & m_{2 g, 2 g}
\end{array}\right)+\left(\begin{array}{c}
-b_{1} \\
\vdots \\
-b_{g} \\
a_{1} \\
\vdots \\
a_{g}
\end{array}\right)\left(a_{1} \cdots a_{g} b_{1} \cdots b_{g}\right)
$$

We note that $\mathbb{Z}[H]$ is an integral domain. By comparing (3.1) with (3.2), we arrive at the following equation.

$$
\left(\begin{array}{ccc}
m_{1,1} & \cdots & m_{1,2 g} \\
\vdots & \ddots & \vdots \\
m_{2 g, 1} & \cdots & m_{2 g, 2 g}
\end{array}\right)\left(\begin{array}{c}
-b_{1} \\
\vdots \\
-b_{g} \\
a_{1} \\
\vdots \\
a_{g}
\end{array}\right)=\left(\begin{array}{c}
-b_{1} \\
\vdots \\
-b_{g} \\
a_{1} \\
\vdots \\
a_{g}
\end{array}\right)
$$

Hence we can conclude

$$
\bar{r}(\varphi) v=v
$$

This completes the proof.

We define the matrix $P_{1}$ as follows. First, we consider the following $2 g$ elements

$$
\gamma_{j}^{\prime}=\left[\beta_{g}, \alpha_{g}\right] \cdots\left[\beta_{j}, \alpha_{j}\right], \quad \gamma_{g+j}^{\prime}=\beta_{j} \quad(j=1, \ldots, g),
$$

and set

$$
P_{1}=\mathfrak{a}\left(\overline{\frac{\partial \gamma_{j}^{j}}{\partial \gamma_{i}}}\right)_{i, j}=\left(\begin{array}{cc}
P_{11} & 0 \\
P_{21} & I_{g}
\end{array}\right)
$$


where

$$
P_{11}=-\left(\begin{array}{cccc}
b_{1} & & & 0 \\
b_{2} & b_{2} & & \\
\vdots & \vdots & \ddots & \\
b_{g} & b_{g} & \cdots & b_{g}
\end{array}\right), \quad P_{21}=\left(\begin{array}{cccc}
a_{1} & & & 0 \\
a_{2} & a_{2} & & \\
\vdots & \vdots & \ddots & \\
a_{g} & a_{g} & \cdots & a_{g}
\end{array}\right)
$$

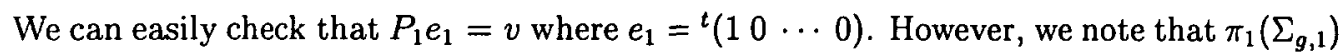
is not generated by $\gamma_{j}^{\prime}$, and also that $P_{1}^{-1}$ is not contained in $G L\left(2 g ; \mathbb{Z}\left[x_{i}{ }^{ \pm 1}, y_{i}^{ \pm 1}\right]\right)$ but $G L\left(2 g ; \mathbb{Z}\left[x_{i}^{ \pm 1}, y_{i}^{ \pm 1}, 1 /\left(1-y_{i}\right)\right]\right)$. Therefore we have the following.

Corollary 3.2. For any element $\varphi \in \mathcal{I}_{g, 1}$, the non-singular matrix $P_{1}$ satisfies the equation

$$
P_{1}^{-1} \bar{r}(\varphi) P_{1}=\left(\begin{array}{c|c}
1 & \rho_{a}(\varphi) \\
\hline 0 & \\
\vdots & \rho_{A}(\varphi) \\
0 &
\end{array}\right)
$$

The Burau representation is the direct sum of its 1-dimensional trivial subrepresentation and the quotient representation. The Gassner representation has the same property. However, The Magnus representation of the Torelli group does not have this property. In fact, we can deduce the following result.

PROPOSITION 3.3. The Magnus representation of the Torelli group is not completely reducible.

ProOF: To get a contradiction, suppose that $\bar{r}$ is completely reducible. Then there exists a non-singular matrix $U$ such that

$$
U^{-1}\left(\begin{array}{c|c}
1 & \rho_{a}(\varphi) \\
\hline 0 & \\
\vdots & \rho_{A}(\varphi) \\
0 &
\end{array}\right) U=\left(\begin{array}{c|ccc}
1 & 0 & \cdots & 0 \\
\hline 0 & & \\
\vdots & & \rho_{A}^{\prime}(\varphi) \\
0 & &
\end{array}\right) .
$$

The coefficients of $U$ belong to a field including $\mathbb{Z}[H]$. By setting

$$
U=\left(\begin{array}{c|c}
U_{1} & U_{2} \\
\hline U_{3} & U_{4}
\end{array}\right)
$$

the equation (3.3) implies

$$
\begin{aligned}
\rho_{a}(\varphi) U_{3} & =0 \\
\rho_{A}(\varphi) U_{4} & =U_{4} \rho_{A}{ }^{\prime}(\varphi)
\end{aligned}
$$




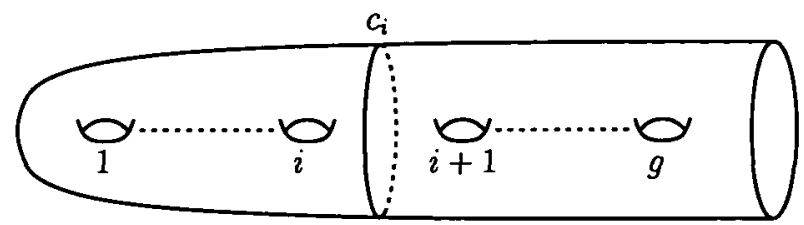

Figure 2: Simple closed curve $c_{i}$

On the other hand, we set $\varphi_{i}$ and $\nu_{i}$ are the Dehn twists about simple closed curves $c_{i}$ and $n_{i}$ as depicted in Figure 2 and Figure 3 respectively and by direct calculation we have

$$
\begin{aligned}
& \varphi_{i}\left(\alpha_{j}\right)=\left\{\begin{array}{ll}
\delta^{-1} \alpha_{j} \delta & j \leqslant i \\
\alpha_{j} & j>i
\end{array} \quad \varphi_{i}\left(\beta_{j}\right)= \begin{cases}\delta^{-1} \beta_{j} \delta & j \leqslant i \\
\beta_{j} & j>i\end{cases} \right. \\
& \nu_{i}\left(\alpha_{j}\right)= \begin{cases}\alpha_{i} \beta_{i}^{-1} \alpha_{i+1} \beta_{i+1} \alpha_{i+1}^{-1} & j=i \\
\alpha_{i+1} \beta_{i+1}^{-1} \alpha_{i+1}^{-1} \beta_{i} \alpha_{i+1} & j=i+1 \\
\alpha_{j} & \text { otherwise }\end{cases} \\
& \nu_{i}\left(\beta_{j}\right)= \begin{cases}\alpha_{i+1} \beta_{i+1}^{-1} \alpha_{i+1}^{-1} \beta_{i} \alpha_{i+1} \beta_{i+1} \alpha_{i+1}^{-1} & j=i \\
\beta_{j} & \text { otherwise }\end{cases} \\
& \nu_{i}^{-1}\left(\alpha_{j}\right)= \begin{cases}\alpha_{i} \alpha_{i+1} \beta_{i+1}^{-1} \alpha_{i+1}^{-1} \beta_{i} & j=i \\
\beta_{i}^{-1} \alpha_{i+1} \beta_{i+1} & j=i+1 \\
\alpha_{j} & \text { otherwise }\end{cases} \\
& \nu_{i}^{-1}\left(\beta_{j}\right)= \begin{cases}\beta_{i}^{-1} \alpha_{i+1} \beta_{i+1} \alpha_{i+1}^{-1} \beta_{i} \alpha_{i+1} \beta_{i+1}^{-1} \alpha_{i+1}^{-1} \beta_{i} & j=i \\
\beta_{j} & \text { otherwise }\end{cases}
\end{aligned}
$$

Here $\delta=\left[\alpha_{1}, \beta_{1}\right]\left[\alpha_{2}, \beta_{2}\right] \cdots\left[\alpha_{i}, \beta_{i}\right]$. Thus we obtain

$$
\begin{aligned}
& \rho_{a}\left(\varphi_{i}\right)=(\underbrace{0, \ldots, 0}_{g-1}, b_{1}, \ldots, b_{i}, \underbrace{0, \ldots, 0}_{g-i}) i=1, \ldots, g \\
& \rho_{a}\left(\nu_{i} \varphi_{i} \nu_{i}^{-1}\right)= \\
& (\underbrace{0, \ldots, 0}_{i-1},-b_{i} b_{i+1}, \underbrace{0, \ldots, 0}_{g-i-1}, b_{1}, \ldots, b_{i-1}, p_{i}, \underbrace{0, \ldots, 0}_{g-i} i=1, \ldots, g-1
\end{aligned}
$$

where $p_{i}=\bar{y}_{i+1}\left(1-\bar{y}_{i}\right)\left(\bar{y}_{i}+\bar{y}_{i+1}-\bar{y}_{i} \bar{y}_{i+1}\right)$. Since we have the equation (3.4) for any element $\varphi \in \mathcal{I}_{g, 1}, U_{3}$ is a zero column vector. Then we $g e t \operatorname{det} U=U_{1} \cdot \operatorname{det} U_{4} \neq 0$ and it implies that $U_{4}$ is a non-singular matrix. Therefore, by (3.5),

$$
\rho_{A}{ }^{\prime}(\varphi)=U_{4}^{-1} \rho_{A}(\varphi) U_{4}
$$




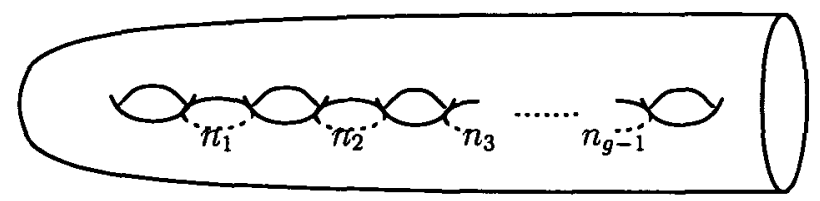

Figure 3: Simple closed curve $n_{i}$

This means that $\rho_{A}^{\prime}$ is conjugate to $\rho_{A}$. By explicit calculation we get $\rho_{A}\left(\tau_{\zeta}\right)=I_{2 g-1}$. Thus we can conclude $\rho_{A}{ }^{\prime}\left(\tau_{\zeta}\right)=I_{2 g-1}$ and $\tau_{\zeta} \in$ ker $\bar{r}$. However, we can check that $\tau_{\zeta}$ is not an element of ker $\bar{r}$. This is a contradiction.

\section{REDUCIBLITY OF THE QUOTIENT REPRESENTATION $\rho_{A}$}

The quotient representation $\rho_{A}$ is irreducible for $g=1$. However, for higher genera, the quotient representation $\rho_{A}$ is reducible. That is, we have the following proposition.

PROPOSITION 4.1. The representation $\rho_{A}$ has a $(2 g-2)$-dimensional subrepresentation $\rho_{B}$ for $g \geqslant 2$.

Proof: We have defined the matrix $P_{1} \in G L(2 g ; R)$ such that

$$
P_{1}^{-1} \bar{r}(\varphi) P_{1}=\left(\begin{array}{c|c}
1 & \rho_{a}(\varphi) \\
\hline 0 & \\
\vdots & \rho_{A}(\varphi) \\
0 &
\end{array}\right)
$$

We remark that $\rho_{A}$ is a homomorphism and that $\rho_{a}$ is a crossed homomorphism. That is to say, for any elements $\varphi, \dot{\psi} \in \mathcal{I}_{g, 1}$

$$
\rho_{A}(\varphi \psi)=\rho_{A}(\varphi) \rho_{A}(\psi), \quad \rho_{a}(\varphi \psi)=\rho_{a}(\psi)+\rho_{a}(\varphi) \rho_{A}(\psi)
$$

Let $\tau_{\zeta}$ be the Dehn twist along a simple closed curve on $\Sigma_{g, 1}$ which is parallel to the boundary as before. Since

$$
\rho_{A}\left(\tau_{\zeta}\right)=I_{2 g-1}, \quad \rho_{a}\left(\tau_{\zeta}\right)=(\underbrace{0, \ldots, 0}_{g-1}, b_{1}, \ldots, b_{g}),
$$

we have

$$
\begin{aligned}
\rho_{a}\left(\tau_{\zeta} \varphi\right) & =\rho_{a}(\varphi)+\rho_{a}\left(\tau_{\zeta}\right) \rho_{A}(\varphi) \\
\rho_{a}\left(\varphi \tau_{\zeta}\right) & =\rho_{a}\left(\tau_{\zeta}\right)+\rho_{a}(\varphi) \rho_{A}\left(\tau_{\zeta}\right) \\
& =\rho_{a}\left(\tau_{\zeta}\right)+\rho_{a}(\varphi) .
\end{aligned}
$$


We recall $\tau_{\zeta} \varphi=\varphi \tau_{\zeta}$ for any element $\varphi \in \mathcal{I}_{g, 1}$, because $\tau_{\zeta}$ is central in $\mathcal{M}_{g, 1}$. Then we get

$$
\begin{gathered}
\rho_{a}\left(\tau_{\zeta}\right) \rho_{A}(\varphi)=\rho_{a}\left(\tau_{\zeta}\right) \\
{ }^{t} \rho_{A}(\varphi)^{t} \rho_{a}\left(\tau_{\zeta}\right)={ }^{t} \rho_{a}\left(\tau_{\zeta}\right)
\end{gathered}
$$

This means that ${ }^{t} \rho_{a}\left(\tau_{\zeta}\right)$ is an eigenvector of the matrix ${ }^{t} \rho_{A}(\varphi)$ with eigenvalue 1 for any $\varphi \in \mathcal{I}_{g, 1}$. Therefore there exists a non-singular $(2 g-1) \times(2 g-1)$-matrix $Q_{1}$ such that

$$
\begin{aligned}
& Q_{1}^{-1}{ }^{t} \rho_{A}(\varphi) Q_{1}=\left(\begin{array}{c|c}
1 & * \\
\hline 0 & \\
\vdots & { }^{t} \rho_{B}(\varphi) \\
0 &
\end{array}\right) \\
& { }^{t} Q_{1} \rho_{A}(\varphi){ }^{t} Q_{1}^{-1}=\left(\begin{array}{c|ccc}
1 & 0 & \ldots & 0 \\
\hline * & & & \rho_{B}(\varphi) \\
* & & &
\end{array}\right) .
\end{aligned}
$$

By putting $Q={ }^{t} Q_{1}{ }^{-1} \cdot Q_{2}{ }^{-1}$, where

$$
Q_{2}=\left(\begin{array}{ccccc}
0 & 1 & 0 & 0 & 0 \\
0 & 0 & 1 & 0 & 0 \\
\vdots & & \ddots & \ddots & \\
0 & 0 & 0 & 0 & 1 \\
1 & 0 & 0 & 0 & 0
\end{array}\right)
$$

we obtain

$$
Q^{-1} \rho_{A}(\varphi) Q=\left(\begin{array}{lll|l} 
& & \\
\multicolumn{3}{|c|}{\rho_{B}(\varphi)} & * \\
& & & \\
\hline 0 & \cdots & 0 & 1
\end{array}\right)
$$

This equation means that the representation $\rho_{A}$ has a $(2 g-2)$-dimensional subrepresentation $\rho_{B}$. This completes the proof.

REMARK 4.2. We define a non-singular matrix $Q_{1}$ by

$$
Q_{1}=\left(\begin{array}{cc}
0 & I_{g-1} \\
-P_{11} & 0
\end{array}\right)
$$

Then we can easily check that $Q_{1} e_{1}={ }^{t} \rho_{a}\left(\tau_{\zeta}\right)$ where $e_{1}={ }^{t}\left(\begin{array}{llll}1 & 0 & \cdots & 0\end{array}\right)$. In this way we can construct $(2 g-1) \times(2 g-1)$-matrices $Q_{1}$ and $Q$, which appear in Proposition 4.1, explicitly. 


\section{IRREDUCIBILITY OF THE REPRESENTATION $\rho_{B}$}

Combining Corollary 3.2 and Proposition 4.1, we obtain the following main result of this paper.

Theorem 5.1. For $g \geqslant 2$ there exsists a non-singular matrix $P \in G L(2 g ; R)$ such that for any element $\varphi \in \mathcal{I}_{g, 1}$

$$
P^{-1} \bar{r}(\varphi) P=\left(\begin{array}{c|ccc|c}
1 & & * & * \\
\hline 0 & & & \\
\vdots & & \rho_{B}(\varphi) & * \\
& & & & \\
\cline { 2 - 5 } & 0 & \cdots & 0 & 1
\end{array}\right) .
$$

Moreover,

$$
\rho_{B}: \mathcal{I}_{g, 1} \longrightarrow G L(2 g-2 ; R)
$$

is a $(2 g-2)$-dimensional irreducible representation of $\mathcal{I}_{g, 1}$.

We have only to set $P=P_{1} \cdot\left(I_{1} \oplus Q\right)$ to obtain the above decomposition. It remains to prove the irreduciblity of $\rho_{B}$.

Let $\mathbb{C}^{n}$ be the $n$ dimensional complex vector space consisting of column vectors. We denote by ${ }^{t} \mathbb{C}^{n}$ the transposed vector space consisting of row vectors. A matrix $X \in M(n ; \mathbb{C})$ is called a pseudoreflection if $X-I_{n}$ has rank 1. If $X$ is a pseudoreflection, then

$$
X=I_{n}-A B
$$

for some $A \in \mathbb{C}^{n}$ and $B \in{ }^{t} \mathbb{C}^{n}$.

TheOREM 5.2. (Formanek [4]) Let $X_{1}=I_{n}-A_{1} B_{1}, \ldots, X_{n}=I_{n}-A_{n} B_{n}$ be $n$ invertible pseudoreflections in $M(n ; \mathbb{C})$, where $n \geqslant 2$. Let $\Gamma$ be the directed graph whose vertices are $1,2, \ldots, n$, and which has a directed edge from $i$ to $j(i \neq j)$ precisely when $B_{i} A_{j} \neq 0$. Let $G$ be the subgroup of $G L(n ; \mathbb{C})$ generated by $X_{1}, \ldots, X_{n}$. Then the following are equivalent.

1. $\operatorname{Span}_{\mathbb{C}}\{G\}=M(n ; \mathbb{C})$

2. For each $i \neq j, 1 \leqslant i, j \leqslant n$, the graph $\Gamma$ contains a directed path from $i$ to $j,\left\{A_{1}, \ldots, A_{n}\right\}$ is a basis for $\mathbb{C}^{n}$ and $\left\{B_{1}, \ldots, B_{n}\right\}$ is a basis for ${ }^{t} \mathbb{C}^{n}$.

3. For each $i \neq j, 1 \leqslant i, j \leqslant n$, the graph $\Gamma$ contains a directed path from $i$ to $j$ and $\operatorname{det}\left(B_{i} A_{j}\right)_{i, j} \neq 0$.

This theorem gives a criterion for a certain representation to be irreducible. This is because, for a group $G^{\prime}$ and a representation $T: G^{\prime} \rightarrow G L(n ; \mathbb{C})$, if $\operatorname{Im} T$ spans $M(n ; \mathbb{C})$, then $T$ is an irreducible representation (see [3]). We shall prove that $\rho_{B}$ is irreducible by making use of this method. 


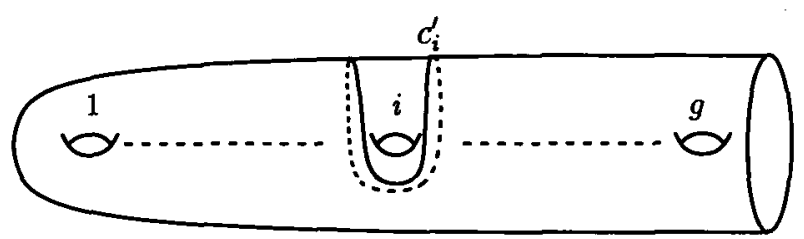

Figure 4: Simple closed curve $c_{i}^{\prime}$

PROOF OF THE IRREDUCIBILITY OF $\rho_{B}$ : Let $\rho_{B(z, w)}$ be the representation

$$
\rho_{B(z, w)}: \mathcal{I}_{g, 1} \longrightarrow G L(2 g-2 ; \mathbb{C})
$$

obtained by specialising $x_{i} \mapsto z_{i}, y_{i} \mapsto w_{i}$ in $\rho_{B}$, where $(z, w)=\left(z_{1}, \ldots, z_{g}, w_{1}, \ldots, w_{g}\right)$ are complex numbers $z_{i} \in \mathbb{C} \backslash\{0\}, w_{i} \in \mathbb{C} \backslash\{0,1\}$. We denote by $L_{g}$ the subgroup of $\mathcal{I}_{g, 1}$ generated by the $2 g-2$ elements $\nu_{1} \varphi_{1} \nu_{1}^{-1}, \varphi_{2}^{\prime}, \nu_{2} \varphi_{2} \nu_{2}^{-1}, \varphi_{3}^{\prime}, \cdots, \nu_{g-1} \varphi_{g-1} \nu_{g-1}{ }^{-1}, \varphi_{g}^{\prime}$. Let us simply write $\psi_{1}, \psi_{2}, \ldots, \psi_{2 g-2}$ for them. Here $\varphi_{i}^{\prime}$ is the Dehn twist about a simple closed curve $c_{i}^{\prime}$ as shown in Figure 4. We note the action of $\varphi_{i}^{\prime}$ on the generators $\gamma_{j}$.

$$
\begin{aligned}
& \varphi_{i}^{\prime}\left(\alpha_{j}\right)= \begin{cases}{\left[\beta_{i}, \alpha_{i}\right] \alpha_{i}\left[\alpha_{i}, \beta_{i}\right]} & j=i \\
\alpha_{j} & \text { otherwise }\end{cases} \\
& \varphi_{i}^{\prime}\left(\beta_{j}\right)= \begin{cases}{\left[\beta_{i}, \alpha_{i}\right] \beta_{i}\left[\alpha_{i}, \beta_{i}\right]} & j=i \\
\beta_{j} & \text { otherwise }\end{cases}
\end{aligned}
$$

Because all $\psi_{i}$ are BSCC maps, that is, the Dehn twists along 0-homologous simple closed curves, the matrices $\rho_{B(z, w)}\left(\psi_{i}\right)$ are pseudoreflections, namely $\rho_{B(z, w)}\left(\psi_{i}\right)=I_{2 g-2}+A_{i} B_{i}$ for some $A_{i} \in \mathbb{C}^{2 g-2}, B_{i} \in{ }^{t} \mathbb{C}^{2 g-2}$. By direct calculation, we get

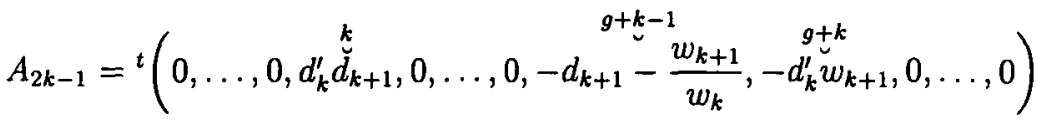

$$
\begin{aligned}
& A_{2 k}={ }^{t}(0, \ldots, 0, \stackrel{g+k}{1}, \stackrel{g+k+1}{-1}, 0, \ldots, 0) \\
& B_{2 k-1}=\left(0, \ldots, 0,-\stackrel{k-1}{w_{k}^{\prime} d_{k+1}^{\prime}},-d_{k}-\stackrel{k}{w_{k}}, 0, \ldots, \stackrel{g+k-1}{w_{k+1}}, 0,-d_{k}^{\prime} d_{k+1}^{\prime}, 0, \ldots, 0\right) \\
& B_{2 k}= \begin{cases}(0, \ldots, 0, \stackrel{k}{\stackrel{k}{1}, \stackrel{k+1}{-1}, 0, \ldots, 0)} & 1 \leqslant k \leqslant g-2 \\
(0, \ldots, 0, \stackrel{g-1}{1}, 0, \ldots, 0) & k=g-1\end{cases}
\end{aligned}
$$


where $d_{k}=1-w_{k}, d_{k}^{\prime}=1-\left(1 / w_{k}\right)$. We remark that $z_{k}$ does not appear in $A_{i}$ and $B_{i}$. Then we have the $(2 g-2) \times(2 g-2)$-matrix $\left(B_{i} A_{j}\right)_{i, j}$ as follows:

$$
\left(B_{i} A_{j}\right)_{i, j}=\left(\begin{array}{cccccccc}
0 & d_{1} d_{2}^{\prime} & & & & & \\
-d_{1}^{\prime} d_{2} & 0 & d_{2}^{\prime} d_{3} & & & & 0 & \\
& -d_{2} d_{3}^{\prime \prime} & 0 & d_{2} d_{3}^{\prime} & & & & \\
& & -d_{2}^{\prime} d_{3} & 0 & d_{3}^{\prime} d_{4} & & & \\
& & & -d_{3} d_{4}^{\prime} & 0 & & & \\
& & & & & \ddots & & \\
& 0 & & & & & 0 & d_{g-1} d_{g}^{\prime} \\
& & & & & & -d_{g-1}^{\prime} d_{g} & 0
\end{array}\right) .
$$

Thus we obtain

$$
\operatorname{det}\left(B_{i} A_{j}\right)=\frac{\left(1-w_{1}\right)^{2}\left(1-w_{2}\right)^{4} \cdots\left(1-w_{g-1}\right)^{4}\left(1-w_{g}\right)^{2}}{w_{1} w_{2}^{2} \cdots w_{g-1}{ }^{2} w_{g}}
$$

and the associated graph can be expressed as

$$
123 \quad 2 g-3 \quad 2 g-2
$$

Therefore by virture of Theorem 5.2 , the representation $\left.\rho_{B(z, w)}\right|_{L_{g}}$ is irreducible. Hence $\rho_{B(z, w)}$ and $\rho_{B}$ are irreducible.

REMARK 5.3. If there exists a single $(z, w)$ such that $\left.\rho_{B(z, w)}\right|_{L_{g}}$ is irreducible, then we can conclude $\rho_{B}$ is irreducible. However, the above proof shows that $\left.\rho_{B(z, w)}\right|_{L_{g}}$ is irreducible for any $z_{i} \in \mathbb{C} \backslash\{0\}$ and $w_{i} \in \mathbb{C} \backslash\{0,1\}$.

\section{SOME REMARKS AND APPLICATIONS}

This section is devoted to give some remarks and applications of the irreducible decomposition.

Let $\mathcal{I}_{g}$ be the Torelli group of $\Sigma_{g}$ and $\mathcal{I}_{g, *}$ that of $\Sigma_{g}$ relative to the base point $* \in \Sigma_{g}$. We have the following by explicit computations.

$$
\begin{aligned}
& \rho_{B}\left(\tau_{\zeta}\right)=I_{2 g-2} \\
& \rho_{B}\left(\widetilde{\alpha}_{i}\right)=\bar{x}_{i} I_{2 g-2} \\
& \rho_{B}\left(\widetilde{\beta}_{i}\right)=\bar{y}_{i} I_{2 g-2}
\end{aligned}
$$

Here $\widetilde{\alpha}_{i} \in \mathcal{I}_{g, 1}$ (respectively $\widetilde{\beta}_{i} \in \mathcal{I}_{g, 1}$ ) is a product of the Dehn twist along $\alpha_{i+}$ (respectively $\beta_{i+}$ ) and the inverse of the Dehn twist along $\alpha_{i-}$ (respectively $\beta_{i-}$ ) as depicted in Figure 5 (respectively Figure 6). The equation (6.6) means that $\rho_{B}$ factors through $\mathcal{I}_{g, *}$.

$$
\rho_{B}: \mathcal{I}_{g, *} \longrightarrow G L(2 g-2 ; R)
$$




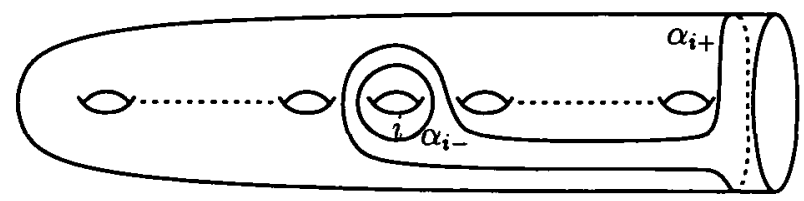

Figure 5: Simple closed curves $\alpha_{i+}$ and $\alpha_{i-}$

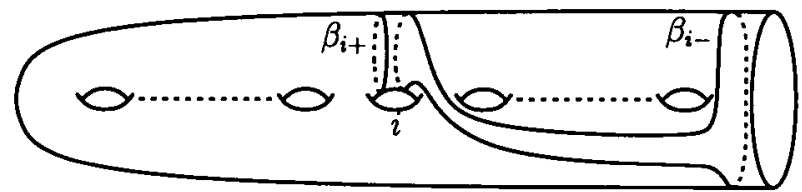

Figure 6: Simple closed curves $\beta_{i+}$ and $\beta_{i-}$

Moreover, the equations (6.6), (6.7) and (6.8) show that $\rho_{B}$ does not factor through $\mathcal{I}_{g}$. However, we write $P G L(2 g-2 ; R)$ for the quotient group of $G L(2 g-2 ; R)$ by all scalar matrices, then we obtain the following representation

$$
\rho_{B}: \mathcal{I}_{g} \longrightarrow P G L(2 g-2 ; R) .
$$

From our previous paper [8], we know that the kernel of the Magnus representation of the Torelli group is non-trivial for $g \geqslant 2$. As an application of the irreducible decomposition, we shall obtain additional information about the kernel. To be more precise we can mention the relation between $\operatorname{ker} \bar{r}$ and the lower central series of $\mathcal{I}_{g, 1}$. We denote by $\mathcal{I}_{(n)}$ the $n$-th term in the lower central series of $\mathcal{I}_{g, 1}$ so that $\mathcal{I}_{(0)}=\mathcal{I}_{g, 1}$ and $\mathcal{I}_{(n)}=\left[\mathcal{I}_{(n-1)}, \mathcal{I}_{(0)}\right]$.

Proposition 6.1. There exists no natural number $n \in \mathbb{N}$ so that

$$
\mathcal{I}_{(n)} \subset \operatorname{ker} \bar{r} .
$$

Proof: We define $f_{n}$ inductively as follows.

$$
f_{1}=\left[\varphi_{1}, \widetilde{\alpha}_{1}\right], f_{2}=\left[f_{1}, \widetilde{\beta}_{1}\right], \ldots, f_{2 k-1}=\left[f_{2 k-2}, \widetilde{\alpha}_{1}\right], f_{2 k}=\left[f_{2 k-1}, \widetilde{\beta}_{1}\right], \ldots
$$

Here $\varphi_{1}, \widetilde{\alpha}_{1}, \widetilde{\beta}_{1}$ are as above. Then $f_{n}$ is an element of $\mathcal{I}_{(n)}$. Now we use the irreducible decomposition. Let $\bar{r}^{\prime}$ be the equivalent representation to $\bar{r}$ so that

$$
\vec{r}^{\prime}(\varphi)=P^{-1} \bar{r}(\varphi) P
$$

By explicit calculation and an inductive proof, we get the following $(i, j)$-components of 
the matrix $\bar{r}^{\prime}\left(f_{n}\right)$.

$$
\begin{aligned}
\bar{r}^{\prime}\left(f_{2 k-1}\right)_{i, j} & = \begin{cases}1 & i=j \\
-\bar{a}_{1}{ }^{k} \bar{b}_{1}{ }^{k-1} & i=1, j=2 \\
-a_{1}{ }^{k} b_{1}{ }^{k-1} & i=g+1, j=2 g \\
0 & \text { otherwise }\end{cases} \\
\bar{r}^{\prime}\left(f_{2 k}\right)_{i, j} & = \begin{cases}1 & i=j \\
-\bar{a}_{1}{ }^{k} \bar{b}_{1}{ }^{k} & i=1, j=2 \\
-a_{1}{ }^{k} b_{1}{ }^{k} & i=g+1, j=2 g \\
0 & \text { otherwise }\end{cases}
\end{aligned}
$$

Thus for any natural number $n \in \mathbb{N}$, we can deduce

$$
f_{n} \notin \operatorname{ker} \bar{r} \text {. }
$$

This completes the proof.

\section{REFERENCES}

[1] M. Abdulrahim, 'Complex specialization of the reduced Gassner representation of the pure braid group', Proc. Amer. Math. Soc. 125 (1997), 1617-1624.

[2] J. Birman, Braids, links and mapping class groups, Annals of Math. Studies No. 82 (Princeton University Press, Princeton N.J., 1975).

[3] L. Dornhoff, Group representation theory Part A (Marcel Dekke Inc., New York, 1971).

[4] E. Formanek, 'Braid group representations of low degree', Proc. London Math. Soc. 73 (1996), 279-322.

[5] D. Johnson, 'A survey of the Torelli group', Contemporary Math. 20 (1983), 165-179.

[6] S. Morita, 'Abelian quotients of subgroups of the mapping class group of surfaces', Duke Math. J. 70 (1993), 699-726.

[7] S. Morita, 'Structure of the mapping class groups of surfaces: a survey and a prospect', in Proceedings of the Kirbyfest (1999), Geom. Topol. Monogr. Vol. 2 (Geom. Topol. Publ., Coventyr), pp. 349-406.

[8] M. Suzuki, 'The Magnus representation of the Torelli group $\mathcal{I}_{g, 1}$ is not faithful for $g \geqslant 2$ ', Proc. Amer. Math. Soc. 130 (2002), 909-914.

Graduate School of Mathematical Sciences

University of Tokyo

3-8-1 Komaba

Meguro

Tokyo 153-8914

Japan

e-mail: macky@ms.u-tokyo.ac.jp 